\title{
INCREMENTAL BOUNDARY VALUE PROBLEMS IN THE PRESENCE OF COUPLING OF ELASTIC AND PLASTIC DEFORMATIONS: A ROCK MECHANICS
ORIENTED THEORY
}

T. Hueckel $\dagger$ and G. MaIER

Department of Structural Engineering, Technical University (Politec.nico), Milan, Italy

(Received 23 February 1976; revised 1 July 1976)

\begin{abstract}
Abetract-In some elastoplastic systems (typically rocklike media) the elastic properties are affected by plastic yielding. This "coupling" phenomenon is dealt with in this paper in the context of the incremental theory of plasticity with nonassociated (lacking normality) flow laws. Some extremum properties and uniqueness conditions are given for solution of the rate problem. Stability conditions are established. The results achieved are equally applicable to media with nonassociative flow rules without coupling, with or without worksoftening, and are believed to be novel in this more traditional area. The results are cast into algebraic formulations for finite element models, in view of their practical applications.
\end{abstract}

\section{INTRODUCTION}

Figure la shows the stress $\sigma$ versus strain $\epsilon$ plot obtained experimentally for a cylinder of rock, specifically sandstone [1], subjected to cyclic uniaxial compression by means of a stiff (displacement controlling) testing machine. The possible presence of lateral pressure strongly affects the main features of the compression test. In fact, the greater such a pressure, the smaller is the inclination of the sloping-down portion of the plot; for large pressure the stress-strain curve is always increasing up to failure as in Fig. 1b [2].

A broad experimental basis indicates that rock behavior can be idealized as elastic-plastic, in the sense that it is substantially time-independent and exhibits significant irreversible strains when sufficiently high "equivalent stress" levels are attained [1-3].

However, the possible presence of the following three main features makes the mechanical response of rock-like media distinct from that of metals and need some generalizations of the classical plasticity theory in order to be accomodated in its framework: (i) an unstable phase may intervene in the post-elastic range; it corresponds to the sloping-down branch of the plot of Fig. la and is usually referred to as strain-softening; (ii) the inelastic strains generated in an incremental process form a vector which need not to be directed as the outward normal vector to the current yield surface; this circumstance occurs for a broad class of materials with internal friction (Coulomb's), such as most soils, and is referred to in the literature by the adjective non-associative attributed to the plastic flow (or incremental) laws; (iii) the apparent elastic stiffness may change drastically as inelastic strains develop; the decreasing slopes of the unloading paths in Fig. 1a illustrate and give experimental evidence to this phenomenon, which will be called herein elastoplastic coupling.

Figure 2 shows a typical idealized description of the behavior of an isotropic rock (such as sandstone) in plane strain, in terms of the principal stresses $\sigma_{\mathrm{I}}$ and $\sigma_{\mathrm{II}}$ (compression positive) in the plane in which deformations are permited ( $\sigma_{\mathrm{m}}$ and $\epsilon_{\mathrm{mI}}=0$ being normal to that plane). The initial yield locus $\phi=0$ is depicted by the heavy solid curve. The straight lines $c$ represent the locus of instantaneous perfectly plastic behavior, i.e. of the "critical or transition states" in the terminology customary in rock mechanics.

A dotted line represents a stress path internal to the acute angle formed by the straight lines $c$; in this case, during the yielding incremental process hardening behavior is exhibited alike to that shown in Fig. 1b for a compression tests in the presence of high transversal pressure: the yield surface expands at yielding without changing its shape (isotropic hardening) as schematically illustrated in Fig. 2 (the light solid line denotes the new yield locus).

tOn leave of absence from Polish Academy of Sciences, I.P.P.T. Warsaw, Poland. 

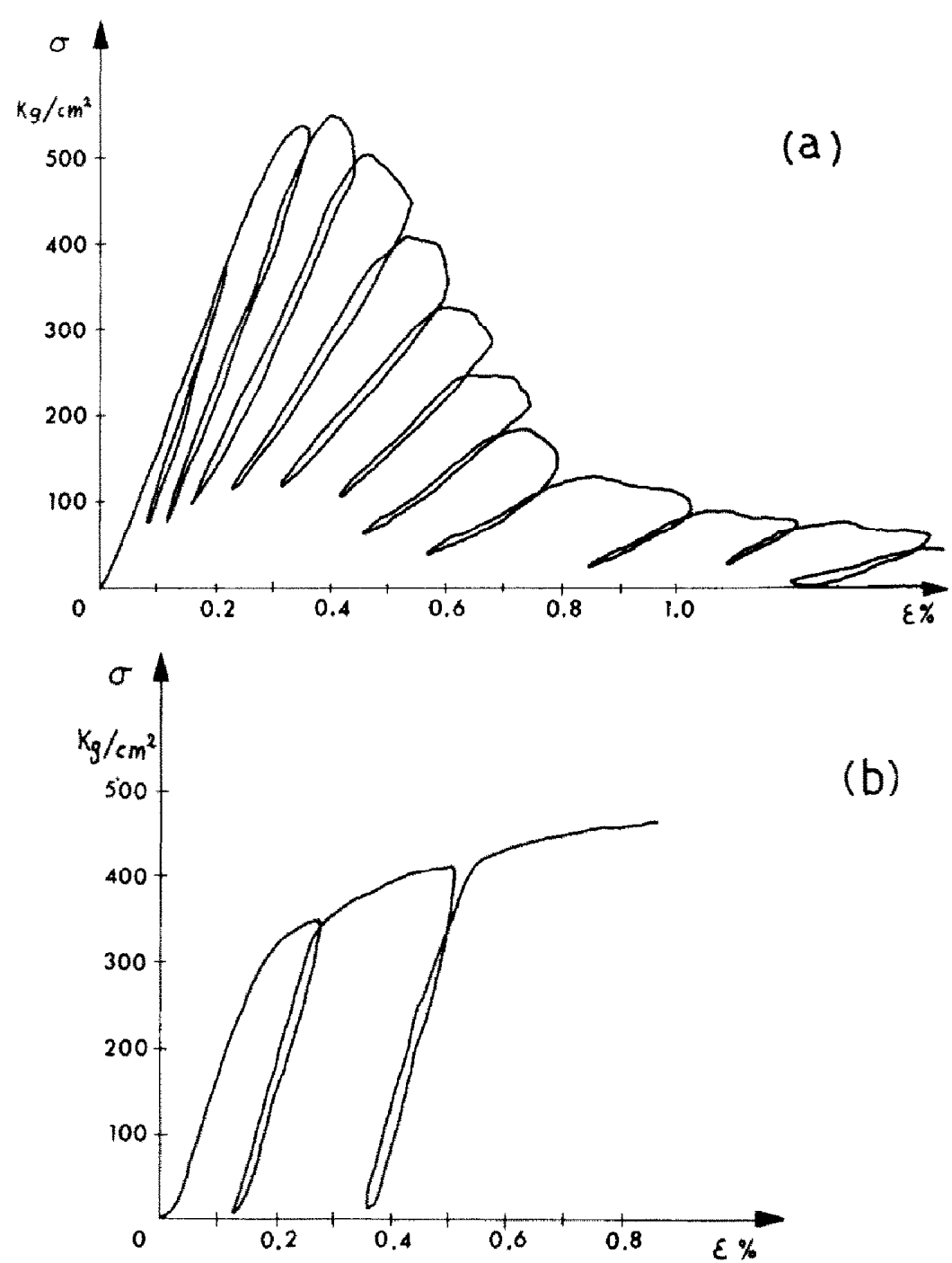

(b)

Fig. 1. Typical compression tests on rock specimens: (a) uniaxial compression, with strong elastoplastic coupling and softening effects; (b) compression in the presence of lateral pressure.

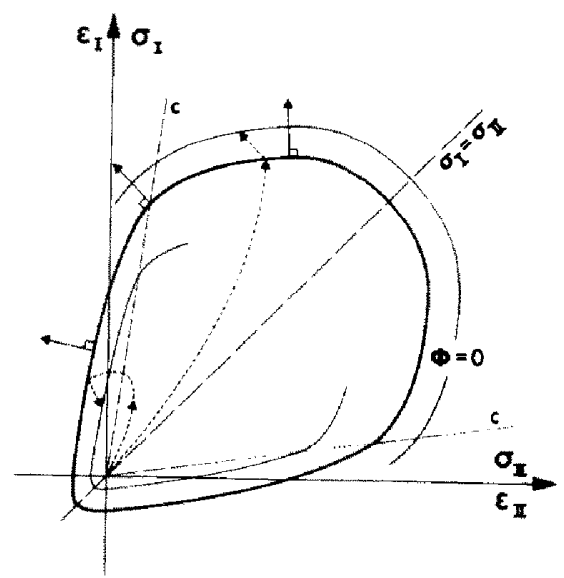

Fig. 2. A typical description of rock behaviour for plane strain stuations.

Another dotted line in Fig. 2 represents a stress path which ends at a yield point outside the acute angle formed by the transition straight lines $c$; in this case the elastic plastic incremental process is accompanied by unstable softening behavior and by shrinking yield surface (like in the compression test of Fig. 1a), as schematically shown in Fig. 2 by the light solid line internal to the 
original yield locus. Softening behavior implies complicate localized phenomena of brittle fracture, but these are usually spread over a region, and incorporated in the concept of material constitutive law in the sense of continuum mechanics, so that workable methods can be set up for the analysis of engineering systems.

Experimental evidence corroborates the customary assumption that the changes of the yield surface are governed by the plastic volumetric deformation.

If associative plastic flow rules are assumed, hardening behavior (within the critical lines $c$ ) is accompanied by plastic consolidation, softening (outside $\hat{c} \hat{c}$ ) by plastic dilatation, perfectly plastic or critical behavior (on $c$ ) by plastic incompressibility. This is shown by Fig. 2 where some outward normal vectors to the initial yield surface are indicated; in fact, incremental volumetric deformations require a non zero component of the strain increment vector along the dashed line $\sigma_{\mathrm{I}}=\sigma_{\mathrm{II}}$. The normality rule which implies the association between plastic strain rates and yield surface is often not in agreement with experimental observations on rocks, so that recourse to nonassociated incremental laws is desirable.

Experiments indicate that the plastic volumetric deformation can be also regarded as the main factor which governs the changes of elastic moduli (or the elastoplastic "coupling") in rocklike media. The shear Kirchhoff modulus appears to be particularly sensitive and is drastically reduced by the plastic dilatation [1,4]. On the basis of the above outlined observational background, various generalizations of the classical plasticity theory were successfully proposed in order to analyse the overall response to loads of engineering systems of nonmetallic materials, such as granular, porous and soil media, (see e.g. [5-9]). However, elastoplastic coupling was investigated only at the level of the material constitutive laws [10,11]; not yet, to the authors' knowledge, at the level of the analysis of continua.

The main scope of this paper is to provide some insight into the influence of elastoplastic coupling on the overall behaviour of inelastic media (specifically rock systems) and to establish a basis for practical methods of analysis capable to deal with this feature of local deformability.

The coupling effects in incremental terms are first recognized to be ameneable to the notion of nonassociative flow rules of plasticity.

For the solutions of the rate boundary value problems, some extremum properties and uniqueness conditions are derived. Some a priori sufficient conditions are established for the overall stability, understood as nonnegativeness of the second-order work performed in any compatible geometric perturbation.

Simple finite element discrete models are considered thereafter, in order to re-formulate in algebraic (matrix) terms the results obtained for continua in tensor field description. On this basis the potential practical use of the results achieved are pointed out and some computational aspects are discussed.

\section{Notation}

For the description of continua in terms of tensor fields the summation convention for repeated indices is adopted. Where finite element models are referred to, matrices (and vectors) are indicated by bold symbols, a tilde means transpose, vector inequalities apply componentwise, 0 denotes a matrix all entries of which are zero.

\section{INCREMENTAL ELASTOPLASTIC CONSTITUTIVE LAWS WITH COUPLING}

Let $\epsilon_{i j}(i, j=1,2,3)$ indicate the tensor of ("small") strains conceived as the sum of an elastic $\left(\epsilon_{i j}^{i}\right)$ and a plastic $\left(\epsilon_{i}^{i}\right)$ part.

Let $\sigma_{h k}$ denote the stress tensor and $C_{i j k}$ the elastic compliance tensor (with the usual symmetry properties); then:

$$
\epsilon_{i j}^{e}=C_{i j k k}\left(\sigma_{i j}, \epsilon_{i j}^{p}\right) \sigma_{h k}
$$

The dependence of compliances $C_{i j h}$ on stresses allows for nonlinear behaviour in the elastic range; tensor $C_{i j h k}$ is assumed positive definite and, hence, the elastic behaviour will always exhibit stability and uniqueness "in the small" in Drucker's sense [14]. The dependence of elastic compliances on plastic strains embodies the coupling effect mentioned in the Introduction.

Considering an incremental elastic-plastic process (Fig. 3) and marking rates by dots, we may 


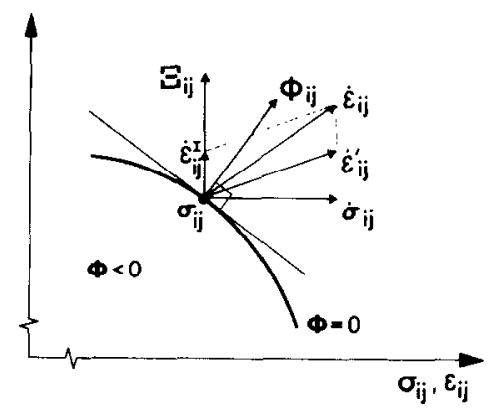

Fig. 3. Schematic representation of multi-component nonassociated flow rules with elastic-plastic coupling for hardening behaviour $(H \geqslant 0)$.

write:

$$
\dot{\epsilon}_{i j}=\dot{\epsilon}_{i j}^{p}+\dot{\epsilon}_{i j}^{e}=\dot{\epsilon}_{i j}^{p}+\dot{\epsilon}_{i j}^{\prime}+\dot{\epsilon}_{i j}^{\prime \prime}
$$

In eqn (2.2), the plastic strain rates $\dot{\epsilon}_{i j}^{p}$ are not required to comply generally with the normality rule (nonassociated flow laws), but are uniquely defined in direction as a vector in the $\epsilon_{i j}$ space:

$$
\dot{\epsilon}_{i j}^{p}=\Psi_{i j} \dot{\lambda}
$$

where $\Psi_{i j}=\left(\partial \psi / \partial \sigma_{i j}\right)\left(\sigma_{i j}, \pi\right)$ denotes the gradient of the plastic potential function, which is assumed as regular (at least differentiable) and dependent on the yield history $\pi ; \dot{\lambda}$ is the plastic multiplier or activation rate.

The reversible part $\dot{\epsilon}_{i j}^{\prime}$ of the elastic strain rate tensor corresponds to a hypothetical purely elastic process $(\dot{\lambda}=0)$ and, hence, reads:

$$
\dot{\boldsymbol{\epsilon}}_{i j}^{\prime}=C_{i j h k} \dot{\sigma}_{h k}
$$

The "coupled" or "mixed" term $\dot{\epsilon}_{i j}$ is the irreversible part of the elastic strain rates, consequent to yielding because of the coupling effect:

$$
\dot{\epsilon}_{i j}^{\prime \prime}=\frac{\partial C_{i j h k}}{\partial \epsilon_{r s}^{p}} \sigma_{h k} \dot{\epsilon}_{r s}^{p}
$$

Substituting (2.3) into (2.5) one obtains:

$$
\dot{\epsilon}_{i j}^{\prime \prime}=\Omega_{i j} \dot{\lambda}
$$

where:

$$
\Omega_{i j} \equiv \frac{\partial C_{i j k k}}{\partial \epsilon_{r s}^{p}} \sigma_{h k} \Psi_{r s}
$$

represents the "coupling tensor" which depends on the stress state and the past plastic history $\pi$.

Let $\Phi_{i j} \equiv\left(\partial \phi / \partial \sigma_{i j}\right)\left(\sigma_{i j}, \pi\right)$ denote the gradient of the yield function $\phi$, i.e. of a regular function of stresses and yielding history $\pi$, such that $\phi \leq 0$ defines the current (smooth) elastic domain. The plastic flow rules for a state at the yield locus $(\phi=0)$ can be expressed as follows:

$$
\begin{gathered}
\dot{\phi}=\Phi_{i j} \dot{\sigma}_{i j}-H \dot{\lambda} \\
\dot{\phi} \leq 0, \dot{\lambda} \geq 0, \dot{\phi} \dot{\lambda}=0
\end{gathered}
$$

where:

$$
H \equiv-\frac{\partial \phi}{\partial \epsilon_{i j}^{*}} \Psi_{i j}
$$

can be identified as the hardening modulus. 
It is assumed that:

$$
\Phi_{i j}\left(\Psi_{i j}+\Omega_{i j}\right)>0
$$

i.e. the irreversible strain rate vector is directed towards the exterior of the instantaneous elastic domain.

Relations (2.9) accomodate the inherent nonlinearity of the incremental plastic laws. For a yielding process $(\dot{\lambda}>0)$, the third relation (2.9) requires $\ddot{\phi}=0$ (i.e. the stress point does not loose contact with the yield locus); hence:

$$
\dot{\lambda}=\frac{1}{H} \Phi_{i j} \dot{\sigma}_{i j}
$$

This shows that:

$H>0$ implies hardening in the instantaneous behaviour, i.e. in the $\sigma_{i j}$ space the stress rate vector $\dot{\sigma}_{i j}$ in a yielding process is directed outward the elastic domain (which, therefore, undergoes local expansion);

$H<0$ means softening, i.e. $\dot{\sigma}_{i j}$ is directed inwards and the elastic domain locally shrinks at yielding;

$H=0$ corresponds to the intermediate case of instantaneous perfectly plastic behaviour, with $\dot{\sigma}_{i j}$ contained in the tangent plane of the yield locus, which does not change, whereas the entity of the plastic strain increments is indeterminate.

Summing up the irreversible parts $\dot{\epsilon}_{i j}^{p}$ and $\dot{\epsilon}_{i j}^{\prime \prime}$ of the strain rates, we have:

$$
\dot{\epsilon}_{i j}^{I}=\Xi_{i j} \dot{\lambda}
$$

where

$$
\Xi_{i j}=\Psi_{i j}+\Omega_{i j}
$$

This shows that the elastoplastic coupling effect, in the above interpretation concerning rates, results into an additional irreversible strain rate. This is directed in general neither along the outward normal to the yield locus, nor along the plastic potential gradient. Therefore, even in the presence of associative plastic flow rules $(\phi \equiv \psi)$, the irreversible (i.e. due to yielding) strain rate vector $\dot{\epsilon}_{i j}^{I}$ deviates from the normal to the yield locus. Hence, the rate problem allowing for coupling bears a similarity to the non-associative rate problem.

On the basis of a static stability criterion (nonnegative second order work for all infinitesimal geometric distrubances), it was shown in [11] that the material is stable if and only if $H \geq H_{1}$, $H_{1} \geq 0$ being a critical value of the hardening modulus expressed as:

$$
2 H_{1}=-\Phi_{i j} E_{i j k k} \Xi_{n k}+\left(\Xi_{n k} E_{n k m n} \Xi_{m n} \cdot \Phi_{i j} E_{i j r s} \Phi_{r s}\right)^{1 / 2}
$$

where $E_{i \text { ink }}$ represents the tensor of elastic moduli. Note that $H_{1}=0$ for associative and uncoupled flow rules.

\section{FORMULATION OF THE INCREMENTAL BOUNDARY VALUE PROBLEM}

With reference to space coordinates $x_{i}(i=1,2,3)$ under the "small deformation" hypothesis, the equilibrium rate equations read:

$$
\begin{aligned}
& \frac{\partial \dot{\sigma}_{i j}}{\partial x_{j}}=\dot{X}_{i} \text { in } V \\
& \dot{\sigma}_{i j} n_{j}=\dot{T}_{i} \text { on } S_{T}
\end{aligned}
$$

where: $X_{i}$ denote body forces, acting over the volume $V$ of the system, $T_{i}$ are surface tractions applied on the free part $S_{T}$ of the boundary, the outward normal to which is indicated by $n_{\text {. }}$. 
Geometric compatibility for rates can be expressed as:

$$
\begin{aligned}
& \dot{e}_{i j}=\frac{1}{2}\left(\frac{\partial \dot{u}_{i}}{\partial x_{j}}+\frac{\partial \dot{u}_{i}}{\partial x_{i}}\right) \text { in } V \\
& \dot{u}_{i}=\dot{u}_{i}^{0} \text { on } S_{u}
\end{aligned}
$$

where $u_{i}^{0}$ are displacements imposed on the constrained part $S_{u}$ of the contour; $\dot{e}_{v}$ indicates the rate tensor of total (but "small") strains, i.e. inclusive of stress-induced strains $\dot{\epsilon}_{\|}$and imposed or "initial" strains $\dot{\epsilon}_{i j}^{0}$ (such as of thermal, chemical and metamorphic nature). Consider a situation $\Sigma$ of the system at an instant of a quasistatic history of external actions $\left(X_{i}, T_{i}, u_{i}^{0}\right.$ and $\left.\epsilon_{i j}^{0}\right)$. All the entities which affect the incremental stress-strain relations are supposed to be known at $\Sigma$ : precisely, previous plastic history $\pi$, the current stress field $\sigma_{\eta}$ and, hence, the plastic volume $V_{p}$ where $\phi\left(\sigma_{i j}, \pi\right)=0$. These relations, discussed in Section 2, are re-written below:

$$
\begin{gathered}
\dot{e}_{i j}=\dot{\epsilon}_{i j}^{0}+C_{i h k} \dot{\sigma}_{h k}+\Xi_{i j} \dot{\lambda} \\
\dot{\phi}=\Phi_{i j} \dot{\sigma}_{i j}-H \dot{\lambda} \text { in } V_{p} \\
\dot{\phi} \leq 0 \text { in } V_{p} \\
\dot{\lambda} \geq 0 \text { in } V \\
\phi \dot{\lambda}=0 \text { in } V \\
\dot{\phi} \dot{\lambda}=0 \text { in } V .
\end{gathered}
$$

Equation (3.9) implies $\dot{\lambda}=0$ in the elastic volume $V-V_{p}$, where the yield limit is not attained $(\phi<0)$ in $\Sigma$. The relation set (3.1)-(3.10) governs the response of the body to given infinitesimal increments (or, to within $\delta t$, rates) of external actions.

\subsection{Minimum principle for deformation rates}

\section{EXTREMUM PROPERTIES OF SOLUTIONS}

For the sake of brevity, imposed displacements and strains will not be considered in what follows; the extension of the subsequent developments to $\dot{u}_{i}^{0} \neq 0, \dot{\epsilon}_{i j}^{0} \neq 0$ is straightforward. Hence, the total strain rates $\dot{e}_{i j}$ are identical to the $\dot{\epsilon}_{i j}$ related to stress rates and will be referred to here.

Because of the noted analogy to nonassociative incremental plasticity, the traditional extremum theorems of incremental plasticity $[12,13]$ cannot be expected to hold in the presence of coupling. However, the above formulated b.v. problem can still be transformed into the minimization of a quadratic form subject to linear constraints. In fact, in view of the sign constraints (3.7) and (3.8), the only nonlinear relation (3.10) is equivalent to:

$$
\int_{V p} \dot{\phi} \dot{\lambda} \mathrm{d} V=0
$$

and this, in turn, can be enforced via optimization as:

$$
\min F_{1}\left(\dot{\lambda}, \dot{\sigma}_{i l}\right)=\min \int_{V_{p}}-\dot{\phi} \dot{\lambda} \mathrm{d} V=\min \int_{V_{p}}\left(\dot{\lambda} H \dot{\lambda}-\dot{\lambda} \Phi_{u} \dot{\sigma}_{u}\right) \mathrm{d} V
$$

under the linear constraints represented by (3.1)-(3.9); eqn (3.6) was used to drop the function $\dot{\phi}$ from this formulation.

Stress rates can be substituted for through (3.5). Thus, $E_{u m k}$ being the elastic stiffness tensor, one obtains the following extremum formulation in terms of kinematic quantities alone:

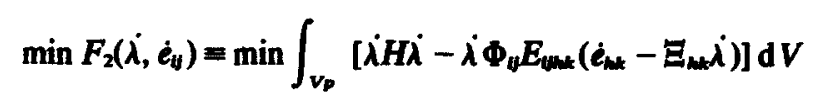

subject to: 


$$
\begin{aligned}
& \text { Incremental boundary value problems } \\
& \left.\dot{e}_{i}=\frac{1}{2}\left(\frac{\partial \dot{u}_{i}}{\partial x_{i}}+\frac{\partial \dot{u}_{i}}{\partial x_{i}}\right) \text { in } V, \dot{u}_{i}=0 \text { on } S_{u}\right) \\
& \frac{\partial}{\partial x_{j}} E_{\| h k}\left(\dot{e}_{h k}-\Xi_{m k} \dot{\lambda}\right)=\dot{X}_{i} \text { in } V \\
& E_{i j k k}\left(\dot{e}_{h k}-\Xi_{h k} \dot{\lambda}\right) n_{j}=\dot{T}_{i} \text { on } S_{T} \\
& \Phi_{l i} E_{i n k}\left(\dot{e}_{n k}-\Xi_{n k} \dot{\lambda}\right)-H \dot{\lambda} \leq 0 \text { in } V_{p} \\
& \dot{\lambda} \geq 0, \phi \dot{\lambda}=0 \text { in } V \text {. }
\end{aligned}
$$

For any fields of kinematic quantities $\dot{e}_{i j}, \dot{\lambda}, \dot{u}_{i}$ complying with (4.13), since both compatibility and equilibrium of the corresponding stresses are fulfilled, the following virtual work equation holds:

$$
\int_{V}\left[\dot{e}_{i} E_{i j k}\left(\dot{e}_{h k}-\Xi_{h k} \dot{\lambda}\right)-\dot{X}_{i} \dot{u}_{i}\right] \mathrm{d} V-\int_{S} \dot{T}_{i} \dot{u}_{i} \mathrm{~d} S=0
$$

Since the left hand side of (4.14) is certainly zero over the feasible domain defined by (4.13), it may be added to the objective function (4.12) without altering the solution of the minimization problem. With this addition, through some algebraic manipulations the objective function can be cast into the following quadratic form, where the new symbol $\dot{\epsilon}_{i j}^{*}$ is defined below by eqn (4.16):

$$
\begin{aligned}
F_{3}=\int_{V_{p}}\left\{\frac{1}{2} \dot{\epsilon}_{i j}^{*} E_{i h k} \dot{\epsilon}_{h k}^{*}+\frac{1}{2} \dot{e}_{i j} E_{i h k} \dot{e}_{h k}+\dot{\lambda}\left[H-\frac{1}{2}\left(\Phi_{l l} E_{i h k} \Phi_{h k}+\Xi_{i j} E_{i j k} \Xi_{h k}\right)\right] \dot{\lambda}\right. \\
\left.-\dot{X}_{i} \dot{u}_{i}\right\} d V-\int_{S_{T}} \dot{T}_{i} \dot{u}_{i} \mathrm{~d} S_{T .} .
\end{aligned}
$$

The functional $F_{3}\left(\dot{\epsilon}_{i j}^{*}, \dot{e}_{i j}, \dot{\lambda}, \dot{u}_{j}\right)$ have to be minimised under the linear constraints (4.13) and, moreover, under the further linear condition

$$
\dot{\epsilon}_{i j}^{*}=\dot{e}_{i j}\left(\Phi_{i j}+\Xi_{i j}\right) \dot{\lambda} .
$$

The interest of this form, in contrast to the bilinear expression (4.12) rests on the fact that a sufficient (not necessary) condition for its convexity is, clearly, that:

$$
H \geq \frac{1}{2}\left(\Phi_{i j} E_{i h k} \Phi_{h k}+\Xi_{i j} E_{i h k} \Xi_{h k}\right) \equiv H^{*}
$$

Though condition (4.17) is very restrictive, it may be useful because of its simplicity and because it guarantees the applicability of algorithms usually more efficient than those for general nonconvex optimization. Note that the above defined $H^{*}$ is larger than the critical value $H_{1}$ (eqn 2.15), whenever $\Phi_{11} E_{\text {thk }} \Xi_{\text {hk }}>0$.

\subsection{Minimum principle for plastic multiplier rates}

The stress rate field can be conceived as the sum of two terms: (i) the fictitious, hypothetically elastic stress response $\dot{\sigma}_{i}^{B}$ to the given external action rates $\dot{T}_{i}, \dot{X}_{i}, \dot{\mu}_{i}^{0}, \dot{\epsilon}_{i j}^{0}$; (ii) the selfstress field $\dot{\sigma}_{i}^{s}$ induced by the (unknown) irreversible strain rate $\dot{\epsilon}_{u}^{L}$ regarded as imposed in purely elastic conditions ("dislocations" in Somigliana-Love sense):

$$
\dot{\sigma}_{u}=\dot{\sigma}_{u}^{E}+\dot{\sigma}_{u}^{s}
$$

The latter stress rate field can be expressed in the form:

$$
\dot{\sigma}_{U}^{2}\left(x_{r}\right)=\int_{V} Z_{u k+k}\left(x_{n} \bar{x}_{r}\right) \Xi_{h k} \dot{\lambda}\left(\bar{x}_{r}\right) \mathrm{d} \bar{V} .
$$

The above tensor-valued, two-point function $Z_{\text {wat }}\left(x_{r}\right.$ and $\bar{x}_{r}$ being the two points and $\mathrm{d} \bar{V}$ an 
infinitesimal volume around the latter) represents the influence function for stresses due to imposed strains. It was constructed and discussed in [8] and turns out to be symmetric and negative semidefinite. $\dagger$

Substituting (4.17) and (4.18) into (4.11), we have:

$$
\min F_{4}(\dot{\lambda}) \equiv \min \int_{V_{p}} \dot{\lambda} H \dot{\lambda} \mathrm{d} V-\iint_{V_{p}} \dot{\lambda}\left(x_{i}\right) U\left(x_{i}, \bar{x}_{i}\right) \dot{\lambda}\left(\bar{x}_{i}\right) \mathrm{d} V \mathrm{~d} \bar{V}-\int_{V_{p}} \dot{\lambda} \Phi_{i j} \dot{\sigma}_{i j}^{E} \mathrm{~d} V
$$

where:

$$
U \equiv \Phi_{i j}\left(x_{r}\right) Z_{i h k}\left(x_{r}, \bar{x}_{r}\right) \Xi_{h k}\left(\bar{x}_{r}\right)
$$

The domain over which the optimization has to be performed is defined as follows by inequalities (3.6) and (3.7) alone, the former being re-written using (4.18), (4.19) and (4.21):

$$
\begin{gathered}
\Phi_{i f} \dot{\sigma}_{i j}^{E}+\int_{V_{p}} U\left(x_{r}, \tilde{x}_{r}\right) \dot{\lambda}\left(\bar{x}_{r}\right) \mathrm{d} \bar{V}-H \dot{\lambda} \leq 0, \text { in } V_{p} \\
\dot{\lambda} \geq 0 \text { in } V_{p} .
\end{gathered}
$$

Equilibrium and compatibility requirements are implicitly fulfilled by virtue of the above definitions of $\dot{\sigma}_{i j}^{E}$ and $\dot{\sigma}_{i j}^{s}$.

The minimization of one of the above functionals, $F_{1}, F_{2}, F_{3}$ or $F_{4}$, over the relevant feasible domain will provide the/a solution of the boundary value problem in point, if and only if the minimum value is zero. In fact in this case alone all governing relations are fulfilled, including (3.10). The formulated minimizations concern quadratic (generally nonconvex) functionals constrained by linear inequalities and equations, and, hence, reduce to tractable quadratic programming problems as soon as a discretization is performed (see Section 7).

The above mathematical optimization problems turn out to be "self-dual", in the sense that their dual problems coincide with the primal. In fact, because of the lack of symmetry of the operators involved, one should not expect pairs of dual extremum principles as in classical plasticity theory [12].

\section{S. UNIQUENESS OF SOLUTION}

If the $\dot{\lambda}\left(x_{r}\right)$ field is known, the whole incremental solution is uniquely defined as in a linear elastic boundary value problem in the presence of imposed strains. In fact, then the inelastic strains can be conceived as imposed strains like in Section 4.2 and the relevant selfstresses follow through (4.19). Suppose that there are two solutions $\dot{\lambda}^{a}$ and $\dot{\lambda}^{b}$ for given increments of boundary conditions and external actions, i.e. for a given elastic rate response $\dot{\sigma}_{i t}^{E}\left(x_{r}\right)$. The left hand side of inequality (4.22) represents an expression of the yield function rates $\dot{\phi}\left(x_{r}\right)$. Writing this expression for $\dot{\lambda}^{a}$ and, subsequently, $\dot{\lambda}^{b}$ and subtracting, one obtains:

$$
\dot{\phi}^{a}-\dot{\phi}^{b}=\int_{V_{p}} U\left(x_{r}, \bar{x}_{r}\right)\left(\dot{\lambda}^{a}-\dot{\lambda}^{b}\right)_{x_{r}} \mathrm{~d} \bar{V}-H\left(\dot{\lambda}^{a}-\dot{\lambda}^{b}\right) .
$$

Multiply both sides of (5.1) by $\dot{\lambda}^{a}-\dot{\lambda}^{b}$, take into account (3.10) for both solutions and integrate over the volume. Thus:

$$
\int_{V_{p}} H\left(\dot{\lambda}^{a}-\dot{\lambda}^{b}\right)^{2} \mathrm{~d} V-\int_{V_{p}}\left(\dot{\lambda}^{a}-\dot{\lambda}^{b}\right)_{x_{r}} U\left(x_{r}, \bar{x}_{r}\right)\left(\dot{\lambda}^{a}-\dot{\lambda}^{b}\right)_{\bar{x}_{r}} \mathrm{~d} V \mathrm{~d} \bar{V}=0
$$

which, compared to (4.20), justifies the following conclusion: if the quadratic part of functional $F_{4}(\dot{\lambda})$ in eqn (4.20) is positive definite, then the rate response of the system is certainly unique. In fact, under this condition eqn (5.2) is satisfied only by $\dot{\lambda}^{a}=\dot{\lambda}^{b}$.

In the sense that, for any imposed strain field $\epsilon_{i j}^{0} \iiint_{V} \epsilon_{i l}^{0}\left(x_{r}\right) Z_{i j k k}\left(x_{n} \tilde{x}_{r}\right) \epsilon_{h k}^{0}\left(\bar{x}_{r}\right) \mathrm{d} V \mathrm{~d} \bar{V} \leqslant 0$. 
This sufficient condition for uniqueness, resting on the extremum property of Section 4.2 , is similar to that given in [8] for nonassociative constitutive laws. Another uniqueness condition, straightforwardly derived from the minimum property of Section 4.1 , is provided by the strict inequality (4.17). In fact, the minimization of a strictly convex functional under linear constraints (hence, over a convex domain) leads to a unique solution, if there is a non-empty feasible domain. In contrast to the previous one, this condition is local (rather than overall) in nature, i.e. it concerns the material behaviour and, hence, is much stronger. In fact, the preceding condition does not rule out $a$ priori any material behaviour, whose lack of uniqueness in the incremental constitutive laws may be compensated for by the connectivity and stiffness of the system.

\section{OVERALL STABILITY}

Following Drucker[14], the continuum considered will be said to be stable in a given equilibrium situation $\Sigma$, if and only if an external agency imposing a kinematically admissible (compatible) infinitesimal geometric disturbance $\dot{u}$, by preserving equilibrium, performs nonnegative (second-order) work whatever the disturbance may be. $\dagger$ Strict stability will be characterized by strictly positive second order work. This static criterion for overall stability can be formulated in the alternative ways:

$$
\begin{gathered}
2 W \equiv \int_{S} \dot{T}_{i} \dot{u}_{i} \mathrm{~d} S+\int_{V} \dot{X}_{i} \dot{u}_{i} \mathrm{~d} V=\int_{V} \dot{\sigma}_{i j}\left(\dot{e}_{i j}\right) \dot{e}_{i j}\left(\dot{u}_{j}\right) \mathrm{d} V \geq 0, \text { for any } \dot{u}_{i} \\
\min W\left(\dot{u}_{i}\right) \geq 0 .
\end{gathered}
$$

In (6.1) or (6.2) the following constraints are implicitly meant to be active: (i) the compatibility eqns (3.3), symbolized by $\dot{\vec{e}}_{i j}\left(\dot{u}_{j}\right)$ in (6.1), and (3.4) with $\dot{u}_{i}^{0}=0$ (homogeneous kinematic boundary conditions), along with the continuity of the velocity field $\dot{u}_{i}$; (ii) the constitutive incremental laws (3.5)-(3.10) with $\dot{\epsilon}_{i j}^{0}=0$, interpreted as defining the dependence $\dot{\sigma}_{i j}\left(\dot{e}_{i j}\right)$; (iii) the equilibrium equations (3.1), (3.2), where the external forces $\dot{X}_{i}$ and $\dot{T}_{i}$ are understood as available variables defined by the equilibrium requirement.

A sufficient and necessary condition for overall stability in the above sense should be derived from the minimization (6.2) subject to all the above constraints, by establishing the requirements to be fulfilled in the considered situation $\Sigma$ for the nonnegativeness of the minimum $W_{m}$. This appears a difficult and impracticable task (although the same approach was successfully adopted for material stability in [11]). Therefore, only sufficient, much stronger, but simple and potentially useful a priori overall stability conditions are derived below from (6.1), (6.2). A priori refers to the fact that quantities pertaining to the situation $\Sigma$, not to the solution by the b.v. incremental problem, are involved.

Overall stability in the sense of (6.1) is clearly implied by local stability, whenever geometric effects on equilibrium relations are negligeable. Hence, an obvious, restrictive (but in the present context not trivial) sufficient condition for stability of the system, is that $H \geq H_{1}$ at any $x_{i}$ (see eqn (2.15) and Ref. [11]), i.e. that the integrand in (6.1) be nonnegative for any $\dot{e}_{i j}$ and, hence, the material be everywhere stable. Practically useful stability conditions are often expressed through the positive semidefiniteness of suitable quadratic forms (of a matrix in discrete cases). In order to achieve this in the present context, the following change will be assumed in the flow rules:

$$
\dot{\phi}=0 \text { in } V_{p}, \quad \dot{\lambda} \text { unconstrained in sign. }
$$

Through the remaining flow rules, (3.5), (3.6) and (3.10), it follows that

$$
\dot{\sigma}_{i j}=B_{i j h k} \dot{e}_{h k}
$$

where:

$$
B_{i j h k} \equiv E_{i j h k}-\left(H-H_{c r}\right)^{-1} E_{i j m n} \Xi_{m n} \Phi_{r s} E_{r s h k}
$$

†It was pointed out in [15], that for frictional systems this condition is not necessary for dynamic stability in Ljapunov sense; it is likely to be sufficient in all cases where the external forces are conservative [20]. 
after having set:

$$
H_{c r}=-\Phi_{h k} E_{h k r s} \Xi_{r s}
$$

It will be assumed henceforth $H>H_{c r}$; in fact, otherwise, as shown in [11], the material behaviour would be not controllable by rigidly imposed deformations (stress "jumps" or "subcritical softening"), i.e. it would exhibit so strong local instability to imply necessarily overall instability. Equation (6.5) defines the elastoplastic tensor, i.e. the moduli which relate stress to strain increments in a yielding process. By virtue of the requirement (6.3), these moduli apply to any process and, hence, the incremental constitutive laws are fully linearized by ignoring purely elastic paths (unloading) throughout the plastic volume $V_{p}$. If $\Xi_{i j}=\Phi_{i j}$ (associative laws without coupling) it is readily seen from eqns (6.5), (6.6) that:

$$
\dot{e}_{i j} E_{i h k} \dot{e}_{h k}-\dot{e}_{i j} B_{i h k} \dot{e}_{h k} \geq 0 \text {, for any } \dot{e}_{h k}
$$

i.e. the second order work for unit volume with the laws modified according to (6.3), bounds from bclow the actual one and, obviously, coincides with it for any $\dot{e}_{i j}$ implying plastic yielding. In other terms, material appears "softer" in elastoplastic incremental processes than in the purely elastic ones. Therefore, if stability in the sense (6.1) is ensured for the modified system, it will be certainly so for the actual one. Unfortunately, inequality (6.7) turns out to be violated for some $\dot{e}_{i j}$ when $\Xi_{i j} \neq \Phi_{i j}$, as in the present theory. In fact, consider the quadratic form which represents the difference (6.7), account taken to eqn (6.5):

where:

$$
\chi \equiv \dot{e}_{i j} \Xi_{i j}^{\prime} \Phi_{r s}^{\prime} \dot{e}_{r s}
$$

$$
\Xi_{i j}^{\prime}=\left(H-H_{c r}\right)^{-1 / 2} E_{i j h k} \Xi_{h k}, \quad \Phi_{i j}^{\prime}=\left(H-H_{c r}\right)^{-1 / 2} E_{j h h k} \Phi_{h k} .
$$

Note that: (a) $\chi$ does not change if one adds to $\dot{e}_{i j}$ a vector $\Delta \dot{e}_{i j}$ normal to the plane of $\Xi_{i j}^{\prime}$ and $\Phi_{i j}^{\prime}$ interpreted as vectors in the $\sigma_{i j}, e_{i j}$-spaces superposed; (b) $\chi=0$ when $\dot{e}_{i j}$ is normal to either $\Xi_{i j}^{\prime}$ or $\Phi_{i j}^{\prime}$; (c) $\chi=$ const are cylinders normal to the plane of $\Xi_{i j}^{\prime}$ and $\Phi_{l j}^{\prime}$, intersecting it in hyperbolae and having as asymptotic planes the planes normal to the vectors $\Xi_{i j}^{\prime}$ and $\Phi_{i j}^{\prime} ;$ (d) $\chi<0$ in the dihedral angle formed by those planes and not containing these vectors; (e) it can be assumed without loss of generality that the Euclidean norms of vectors $\Xi_{i j}^{\prime}$ and $\Phi_{i j}^{\prime}$ are equal; in fact, this can be always achieved by suitably re-defining the yield function $\varphi$. From the above geometric remarks it follows that the minimum $\chi_{m}$ of $\chi$ under the constraint $\dot{e}_{i j} \dot{e}_{i j}=1$ (i.e. over a unit sphere centered on the considered stress point $\sigma_{i j}$ ) is obtained along the bisectrix of the angle between $\Xi_{i j}^{\prime}$ and $-\Phi_{i i}^{\prime}$

$$
\dot{e}_{i j}=\left(\Xi_{i j}^{\prime}-\Phi_{i j}^{\prime}\right)\left[\left(\Xi_{h k}^{\prime}-\Phi_{h k}^{\prime}\right)\left(\Xi_{h k}^{\prime}-\Phi_{h k}^{\prime}\right)\right]^{-1 / 2}
$$

By substituting eqn (6.10) in (6.8):

$$
\chi_{\min }=\left(\Xi_{i j}^{\prime}-\Phi_{i j}^{\prime}\right) \Xi_{i j}^{\prime} \Phi_{h k}^{\prime}\left(\Xi_{h k}^{\prime}-\Phi_{h k}^{\prime}\right)\left[\left(\Xi_{r s}^{\prime}-\Phi_{r s}^{\prime}\right)\left(\Xi_{r s}^{\prime}-\Phi_{r s}^{\prime}\right)\right]^{-1} \leq 0 .
$$

Now, consider the following quadratic form, $\delta_{i j}$ being the Kronecker delta:

having set:

$$
2 W^{*}=\dot{e}_{i j} M_{i j h k} \dot{e}_{h k}
$$

$$
M_{i j k}=\left\{\begin{array}{c}
E_{i m k} \text { in } V-V_{p}(\text { where } \phi<0) \\
B_{i j k}+\frac{1}{2}\left(\delta_{i k} \delta_{j k}+\delta_{u k} \delta_{j h}\right) \chi_{\min }, \text { in } V_{p}
\end{array}\right\}
$$

The scalar $W^{*} \delta t^{2}$ represents a lower bound on the actual second-order work density in the presence of coupling and/or nonnormality, both in the case of elastoplastic loading and elastic unloading. In fact, by the very definition of the tensor $M_{i j h}$ :

$$
\begin{gathered}
\dot{e}_{i j} E_{i j k k} \dot{e}_{h k} \geq \dot{e}_{m n} M_{m n r s} \dot{e}_{r s}, \text { for any } \dot{e}_{i j} \\
\dot{e}_{i j} B_{i j h k} \dot{e}_{h k} \geq \dot{e}_{m n} M_{m n n s} \dot{e}_{r s}, \text { for any } \dot{e}_{i j} .
\end{gathered}
$$


Equality holds in (6.14) when $\dot{e}_{i j}$ takes on the expression (6.10). Therefore, if the fictitious material characterized by the tensor $M_{i j h k}$ (with $\dot{\phi}=0$ and $\dot{i}$ unconstrained) is stable, i.e. if $M_{i j h k}$ is positive semidefinite, the real material will be stable as well.

The positive semidefiniteness of $\boldsymbol{M}_{\text {inh }}$ is, hence, sufficient for local and, consequently, overall stability at the situation $\Sigma$. Note that this condition implies, through eqn (6.15) that also the elastoplastic stiffness $B_{i j h k}$ be positive semidefinite and, hence, that $H \geqslant H_{1}$.

A still restrictive overall condition follows from (6.14), (6.15) through the formulation (6.1). As a matter of fact, the second-order work (to within $\delta t^{2}$ ) for the modified system with the above fictitious material is

$$
W^{*}=\int_{V}\left(\frac{\partial \dot{u}_{i}}{\partial x_{j}}+\frac{\partial \dot{u}_{i}}{\partial x_{i}}\right) M_{i j k}\left(\frac{\partial \dot{u}_{h}}{\partial x_{k}}+\frac{\partial \dot{u}_{k}}{\partial x_{h}}\right) \mathrm{d} V
$$

and the consequent overall stability condition reads:

$$
W^{*} \geq 0, \quad \text { for any } \dot{u}_{i} \text { with } \dot{u}_{i}=0 \text { on } S_{u} \text {. }
$$

The condition (6.17) does not rule out the occurrence of non-definite tensor $\boldsymbol{M}_{\text {thk }}$ locally, which can be compensated for by continuity constraints on $e_{l}$ and by the stiffness of surrounding material. However, it does rule out this occurrence over any finite volume.

\section{ALGEBRAIC FORMULATIONS BASED ON FINITE ELEMENT MODELS}

A typical engineering situation in which elastoplastic coupling effects may be important is the excavation of an opening (e.g. tunnel) in rock. The stress and strain states generated by it and possibly by surface loads, can imply extensive plastic deformations. Such a system may be often conceived as a two-dimensional continuum in plane strains. A frequently adopted discretization rests on its subdivision into triangles, over each of which the displacement field is assumed to be linear, geometric compatibility (displacement continuity) being imposed across the triangle sides (Fig. 4a)[16].

The intrinsic deformation of the $i$-th constant-strain triangular finite element can be described by the vector $q^{i}$ of its three side elongations. The static quantities transmitted to the element by the surrounding environment will be lumped into three pairs of self-equilibrated nodal forces (Fig. 4b). The three component vector $\mathbf{Q}^{l}$ which defines these element forces for unit thickness, corresponds to $\mathbf{q}^{l}$ in the sense that the scalar product $\tilde{\mathbf{Q}}^{i} \delta \mathbf{q}^{i}$ represents the work performed over element $\boldsymbol{i}$ for its infinitesimal deformation $\delta q^{i}$. Henceforth bold-face symbols will represent matrices and column-vectors, a superposed tilde will denote transpose. The variables $\mathbf{q}^{i}$ and $\mathbf{Q}^{i}$ are independent "natural" generalized strains and stresses, respectively, of the $i$-th finite element, the former being unaffected by rigid body motions, the latter being a set of equilibrated

(a)

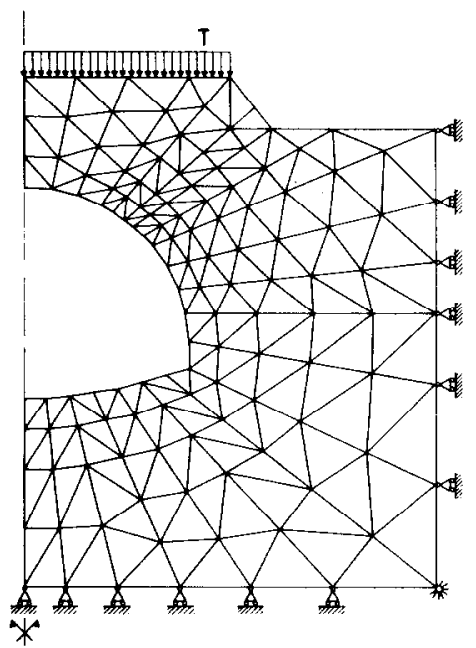

(b)

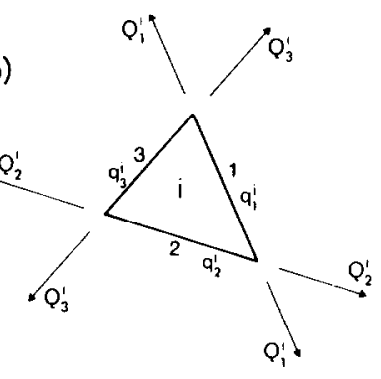

Fig. 4. Finite element discretization of a two-dimensional (plane strain) system (a), and natural generalized variables for a (constant strain) finite element (b). 
forces. By their definitions, $\mathbf{q}^{i}$ is related to the three independent true "engineering strain" components $\left(\epsilon_{11}, \epsilon_{22}\right.$ and $\left.2 \epsilon_{12}\right)$ through a nonsingular linear transformation (for homogeneous elements), $Q^{\prime}$ is related to the corresponding actual stresses in the plane $\left(\sigma_{11}, \sigma_{22}\right.$ and $\left.\sigma_{12}\right)$ through the contragradient transformations:

$$
\mathbf{q}^{i}=\mathbf{T}^{i} \boldsymbol{\epsilon}^{i}, \quad \boldsymbol{\sigma}^{i}=\tilde{\mathbf{T}}^{i} A^{i} \mathbf{Q}^{i}
$$

where matrix $\mathbf{T}^{i}$ depends on the element geometry alone and $A^{i}$ is the area of element $i$. By virtue of eqns (7.1), the material laws (3.5)-(3.10) can be straightforwardly transformed into the relevant "element laws" in terms of $\mathbf{q}^{\mathbf{i}}$ and $\mathbf{Q}^{\prime}$, by preserving all mathematical and mechanical features unaltered. Specifically, with self evident meaning of the symbols, in the $\mathbf{q}^{i}$ and $\mathbf{Q}^{i}$-spaces superposed the element rate laws corresponding to (3.5) and (3.6) read:

$$
\dot{\mathbf{q}}^{i}=\mathbf{E}^{-1 i} \dot{\mathbf{Q}}^{i}+\Xi^{i} \dot{\lambda}^{i}, \quad \dot{\phi}^{i}=\tilde{\boldsymbol{\Phi}}^{i} \dot{\mathbf{Q}}^{i}-H^{i} \dot{\lambda}^{i}
$$

Let $\dot{\lambda}$ and $\dot{\phi}$ denote the vectors which contain as components (taken in the same order) all the scalars $\lambda^{i}$ and $\dot{\phi}^{\prime}$, respectively, for $i=1 \ldots m^{p}, m^{p}$ being the number of the finite elements at the yield limit in the considered situation of the system. Vectors $q$ and $\mathbf{Q}$ will contain as subvectors all $q^{i}$ and $\mathbf{Q}^{i}$, respectively, for all, say $m$, elements in the model. Correspondingly, the $3 \times 3$ elastic stiffness matrices are assembled in the $3 \mathrm{~m} \times 3 \mathrm{~m}$ block-diagonal matrix $\mathbf{E} \equiv \operatorname{diag}\left[\mathbf{E}^{i}\right.$ ]. Let $\mathbf{H} \equiv \operatorname{diag}\left[H^{i}\right]$ for $i=1 \ldots m^{p}$. Vectors $\Xi^{i}$ and $\Phi^{i}$ will form the block-diagonal matrices $\Xi^{*}$ and $\boldsymbol{\Phi}^{*}$,respectively, which, supplemented by null matrices $\mathbf{0}$, provide the $\mathrm{m}^{p} \times 3$ matrices $\tilde{\Xi} \equiv\left[\tilde{\Xi}^{*} \mid \tilde{0}\right]$ and $\tilde{\Phi} \equiv[\tilde{\Phi} * \mid \tilde{0}]$. Using the above symbols, one may write the local deformability laws for the whole (disassembled) model in the following compact matrix form:

$$
\begin{gathered}
\dot{\mathbf{q}}=\mathbf{E}^{-1} \dot{\mathbf{Q}}+\Xi \dot{\lambda}, \quad \dot{\boldsymbol{\phi}}=\tilde{\mathbf{\Phi}} \dot{\mathbf{Q}}-\mathbf{H} \dot{\boldsymbol{\lambda}} \leq \mathbf{0} \\
\dot{\boldsymbol{\lambda}} \geq \mathbf{0}, \quad \dot{\boldsymbol{\phi}} \dot{\boldsymbol{\lambda}}=0 .
\end{gathered}
$$

Any configuration change of the discrete model in point is defined by a vector $\mathbf{u}$ of the, say, $2 n$ displacements at the triangle vertices which do not belong to the fixed part of the boundary. Under the usual small deformation assumption, the compatibility and the equilibrium equations of the system read:

$$
\dot{\mathbf{q}}=\mathbf{C} \dot{\mathbf{u}}, \quad \check{\mathbf{C}} \dot{\mathbf{Q}}=\dot{\mathbf{P}}
$$

where $\mathbf{C}$ is a (full column-rank) matrix depending on the mesh geometry only and $\mathbf{P}$ denote the $2 n$-vector of nodal loads (equivalent, through the discretization process, to given surface and body forces acting on the original continuum).

The relation set (7.3)-(7.5) represents the algebraic counterpart, for the finite element model assumed, to the tensor formulation (3.1)-(3.10) of the rate problem, for $\dot{\epsilon}_{i j}^{0}=0, \dot{u}_{i}^{0}=0$.

In view of numerical applications, the main results obtained for continua in the preceding sections will be straightforwardly re-formulated below on the basis of the above discrete formulation.

The contraints (5.14), if referred to the above discrete models, become:

$$
\left.\begin{array}{r}
\dot{\mathbf{q}}=\mathbf{C} \dot{\mathbf{u}}, \quad \tilde{\mathbf{C}} \mathbf{E}(\dot{\mathbf{q}}-\Xi \dot{\boldsymbol{\lambda}})=\dot{\mathbf{P}} \\
\dot{\mathbf{\Phi}} \mathbf{E}(\dot{\mathbf{q}}-\Xi \dot{\boldsymbol{\lambda}}) \leq \mathbf{H} \dot{\boldsymbol{\lambda}}, \quad \dot{\boldsymbol{\lambda}} \geq \mathbf{0}
\end{array}\right\}
$$

The kinematic rate solution (if there is any) is defined by minimizing (to zero) over the feasible domain (7.6) either:

$$
F_{2}=\frac{1}{2} \dot{\boldsymbol{\lambda}} \mathrm{H} \dot{\lambda}-\dot{\boldsymbol{\lambda}} \mathrm{E}(\dot{\mathrm{q}}-\Xi \dot{\lambda})
$$




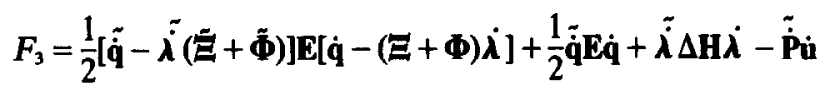

where:

$$
\Delta \mathbf{H} \equiv \mathbf{H}-\frac{1}{2}(\overline{\mathbf{\Phi}} \mathbf{E} \boldsymbol{\Phi}+\check{\Xi} \mathbf{E} \Xi)
$$

$\Delta \mathbf{H}$ represents a diagonal matrix which is positive semidefinite if inequality (4.17) holds for all elements at the yield limit.

In the present algebraic context, the kernel $U$, eqn (4.20), of the second integral in (4.19) becomes a $m^{p} \times m^{p}$ matrix:

$$
\mathbf{U}=\mathbf{\Phi} \mathbf{Z} \Xi
$$

where $\mathrm{Z}$ represents the counterpart to the tensorvalued Green functions (4.18) and is the (symmetric, negative semidefinite) matrix of order $3 m$ which transforms imposed strains into consequent selfstresses for the model supposed linear elastic with the current elastic stiffness. Hence, when the elastic stress rate vector $\dot{\mathbf{Q}}^{B}$ is known, vector $\dot{A}$ which defines the plastic strain rates over the plastic zone can be determined by solving the quadratic programming problem (if its minimum is zero)

subject to:

$$
\text { minimize } \tilde{\dot{\lambda}}(\mathbf{H}-\mathbf{U}) \dot{\boldsymbol{\lambda}}-\tilde{\mathbf{Q}}^{E} \boldsymbol{\Phi} \dot{\boldsymbol{\lambda}}
$$

$$
(\mathbf{H}-\mathbf{U}) \dot{\boldsymbol{\lambda}} \geq \tilde{\boldsymbol{\Phi}} \dot{\mathbf{Q}}^{E}, \quad \dot{\boldsymbol{\lambda}} \geq \mathbf{0}
$$

or, alternatively, by solving the following linear complementarity problem, which is clearly equivalent to (7.11), (7.12):

$$
\begin{aligned}
& (\mathbf{H}-\mathbf{U}) \dot{\lambda} \geq \dot{\Phi}_{\mathbf{Q}}^{B}, \quad \dot{\lambda} \geq 0 \\
& \dot{\boldsymbol{\lambda}}(\mathbf{H}-\mathbf{U}) \dot{\boldsymbol{\lambda}}-\dot{\mathbf{Q}}^{E} \boldsymbol{\Phi} \dot{\boldsymbol{\lambda}}=0 .
\end{aligned}
$$

Uniqueness of the rate response of the model in the considered situation is ensured if matrix $\mathbf{H}-\mathbf{U}$ is positive definite. A stronger sufficient condition for uniqueness is the positive definiteness of matrix $\Delta H$, eqn (7.9). Proofs of these statements are as in Section 5. Let $\mathbf{M}^{d}$ denote the fictitious elastic stiffness matrix for an element, constructed with the criterion of Section 6 , namely, according to (6.13):

$$
\mathbf{M}^{\mathbf{\prime}}=\left\{\begin{array}{c}
\mathbf{E}^{i} \quad \text { for elastic elements } \\
\mathbf{B}^{\prime}+\mathbf{I}_{\chi_{m}}{ }^{\prime} \text { for } \quad i=1 \ldots m^{p}
\end{array}\right\}
$$

where $\chi_{m}{ }^{i}$ is a scalar defined as in (6.9) and (6.14), but on the basis of $E^{i}, \Phi^{\prime}$ and $\Xi^{\prime}$ pertaining to element $i$. Thus, forming the matrix $M \equiv \operatorname{diag}\left[M^{\prime}\right]$, the sufficient condition (6.16), (6.17) for overall stability has the following discrete counterpart:

$$
2 W^{*} \equiv \tilde{\dot{\mathbf{u}}} \tilde{\mathbf{C}} \mathbf{M C} \dot{\mathbf{u}} \geq 0, \quad \text { for all } \mathbf{u}
$$

i.e. it can be expressed by the positive semidefiniteness of the symmetric part of the $n \times n$ matrix $\tilde{\mathbf{C}} \mathbf{M C}=\tilde{\mathbf{C}} \mathbf{M}_{s} \mathbf{C}, \mathbf{M}_{s}$ being the symmetric part of $\mathbf{M}$. Condition(7.16) is slightly less restrictive than the positive semidefiniteness requirement on matrix $\mathbf{M}^{t}$ : it does not rule out unstable behavior of some elements surrounded by stable ones in the sense of eqn (4.1). However, the condition appears to become more stringent as the finite element mesh becomes finer.

From the computational standpoint, the following remarks are of practical importance:

(a) The discretized boundary value problem under consideration can be dealt with by minimizing the quadratic form (7.7) or (7.8) subject to the linear constraints (7.6). Although the objective functions are not necessarily convex, this mathematical programming problems, because of the linearity of the constraints, can be always solved fairly efficiently by algorithms, 
such as that recently proposed in [17]. However, also iterative procedures of Newton-Raphson type, such as "initial stress" method [16], are also applicable and may be computationally competitive.

(b) If matrix $\Delta H$, eqn (7.9), is positive semidefinite, then any traditional technique for convex quadratic programming may be employed [18].

(c) Remarks (a) and (b) hold true also for the extremum formulation (7.11), (7.12), with the additional comment that preliminary linear calculations are required to produce matrix $\mathbf{Z}$ but, as a compensation, the size of the mathematical programming problem is substantically reduced, since there are only as many variables as yielding finite elements.

(d) Formulation (7.13), (7.14) can be processed numerically as a linear complementarity problem by mans of "ad-hoc" algorithms, see e.g. [19], whenever matrix $(\mathbf{H}-\mathbf{U})$ is positive semidefinite.

(e) The uniqueness and overall stability conditions can be of practical value for discrete models since they reduce to numerical checks of positive definiteness of suitable matrices. The former turns out to be less restrictive than the latter.

(f) The evolutive analysis of the system for a given history of external actions can be performed step-by-step by approximate time integration of the rate problem discussed herein. However, it must be noted that the presence of elasto-plastic coupling requires the up-dating from step to step of the matrices which rest on the changing elastic properties (such as $\mathbf{E}, \mathbf{M}, \mathbf{Z}$ ), as in the "tangent modulus" approach to elastoplastic analysis.

(g) For three dimensional systems all the developments of this Sections hold true unaltered, just by re-interpreting the meaning of the symbols $\left(q^{i}\right.$ and $\mathbf{Q}^{i}$ are vectors of natural generalized strains and stresses, respectively, of constant strain tetrahedrical finite elements). But the dramatic increase in the size of the algebraic problem would suggest to make recouse of discrete models more "refined" than those referred to herein.

\section{CONCLUSIONS}

The conclusions substantiated by this paper can be summarized as follows:

(a) Changes of elastic-properties due to plastic deformations (elasto-plastic coupling, typical in rocks and alike media) can be accomodated in the framework of the incremental plasticity theory, making recourse to the notion of nonassociated flow rules.

(b) Elastoplastic coupling, as the often accompanying phenomena of strainsoftening and deviation from normality of the plastic strain rates, may produce material instability in the sense that the second-order work density may become negative.

(c) Local unstable behaviour of materials may entail overall instability of systems even when geometry changes do not affect the equilibrium relations (first-order, small deformation theory). A sufficient condition which guarantees overall stability, in the sense of nonnegative total 2 order work, was established by referring in the plastic zones to a fictitious material with suitably modified, linear incremental constitutive laws.

(d) The solution of the incremental boundary values problem can be achieved by the minimization of quadratic functionals under linear equations and inequalities, if the minimum is zero (otherwise no solution exists). The extremum characterizations of solutions do not arise in pairs as in classical plasticity. The solution uniqueness is guaranteed only under sufficient conditions of local or overall nature, the overall condition being much less stringent than the above sufficient conditions for stability.

(e) By means of finite element discretization numerical solutions of incremental problems in engineering situations can be obtained through available algorithsms of mathematical programming.

Acknowledgements-One of the authors (T.H.) acknowledees with gratitude the support of the Exchange Programme between the National Research Council of Italy and the Polish Academy of Sciences during his stay at the Centre for Computational Structural Mechanics, Department of Structural Engineering, Technical University (Politecnico) of Milan. This research was performed in connection with work (author G.M.) under the E.N.E.L./C.R.I.S. contract No. 30.

\section{REFERENCES}

1. Z. T. Bieniawski, Deformational behaviour of fractured rock under multiaxial compression. Structure, Solid Mech. and Engineering Design, Southampton (Edited by M. Te'eni) Part 1, p. 589-598. Wiley-Interscience, New York (1971).

2. J. M. Edmond and M. S. Paterson, Volume changes during deformation of rocks at high pressures. Int. J. Rock. Mech. Min. Sci. 9, 161-182 (1972). 
3. T. W. Miller andJ. B. Cheatham, Jr, A new yield condition and hardeningrule for rocks. Int. J. Rock Mech. Min. Sci.9, 453-474 (1972).

4. D. Karsan and J. O. Jirsa, Behaviour of concrete under compressive loadings. Proc. ASCE, ST \$5, 2543-2561 (1969).

S. J. B. Burland and X.H. Roscoe, The generalised stress strain behaviour of soft clays. Engineering Plasticity. Cambridge University Press (1968).

6. A.W. Jenike and R. T. Shield, On the plastic flow of Coulombsolids beyond original failure. J. Appl. Mechs 26,599-602 (1959).

7. J. R. Green, A plasticity theory for porous solids. Int. J. Mech. Sci. 14, 215-224 (1972).

8. G. Maier, A minimum principle for incremental elastoplasticity with non-associated flow-laws. J. Mech. Phys. Solids 18, $319-330(1970)$.

9. G. C. Nayak and O.C. Zienkiewicz, Elasto-plastic stress analysis. A generalization for various constitutive relations including strain softening. Int. J. Numerical Methods Engng \$, 113-135 (1972).

10. T. Hueckel, On plastic flow of granular and rocklike materials with variable elasticity moduli. Bull. Acc. Polonaise, Serie Sci. Tech., XXII(8), 405-414 (1975).

11. T. Hueckel and G. Maier, On nonassociated and coupled flow rules of elasto-plasticity for geological media. Int. J. Rock Mech. Min. Sci., to appear.

12. W. T. Koiter, General theorems of elastic-plastic solids. Progress in Solid Mechanics (Edited by I. N. Sneddon and R. Hill), p. 167-221. North Holland, Amsterdam (1960).

13. M. Capurso and G. Majer, Incremental elasto-plastic analysis and quadratic optimization. Meccanica 2, 1-10 (1970).

14. D. C. Drucker, On the postulate of stability of material in mechanics of continua. J. MEcanique 3, 235-249 (1964).

15. J. Mandel, Conditions de stabilite et postulat de Drucker, Rheology and Soil Mechanics, IUTAM Grenoble 194(Edited by J. Kravtchenko and P. M. Sirieys). Springer, Berlin (1966).

16. O. C. Zienkiewicz, The Finite Element Method in Engineering Science. M Graw-Hill, London (1971).

17. M. J. Best and K. Ritter, An accelerate conjugate direction method to solve linearly constrained minimization problems, University of Waterloo, Waterloo, Ontario, Research Repon No. CORR-73-13 (1973).

18. H. P. Künzi and W. Krelle, Nonlinear Programming. Blaisdell, Walthan, Mass., (1966).

19. R. W. Cottle and G. B. Dantzig, Complementary pivot theory of mathematical programming. Mathematics of the Decision Sciences, Part 1, Lectures on Applied Math., Vol. 11 (1958).

20. R. Hill, A general theory of uniqueness and stability in elastic plastic solids. J. Mech. Phys. Sollds 6, 236-249 (1958). 